\title{
Analytical Electron Microscopy Characterization of a Temperature-Stable Relaxor Ferroelectric Ceramic
}

\author{
Teresa Roncal-Herrero $^{1 *}$, John Harrington ${ }^{1}$, Aurang Zeb ${ }^{2}$, Steven J Milne ${ }^{1}$ and Andy P. Brown ${ }^{1}$ \\ ${ }^{1}$ School of Chemical and Process Engineering, University of Leeds, Leeds, United Kingdom. \\ 2.Department of Physics, Islamia College Peshawar, KP, Pakistan. \\ * Corresponding author: t.roncal@leeds.ac.uk
}

There is growing interest in ferroelectric ceramics to develop new capacitor materials that can operate in extreme environments (e.g. aerospace, automotive sector, military and power electronics applications). The chemical composition and structure of the ceramics influence the electrical characteristics of the capacitors. Class II capacitors offer a high volumetric efficiency due to the high relative permittivity, $\varepsilon_{\mathrm{r}}$, of the ceramic dielectric. Materials used for this purpose are in general barium titanate $\left(\mathrm{BaTiO}_{3}\right)$ derivatives with high permittivity values with $\pm 15 \%$ stability over a wide temperature range, but with an upper ceiling temperature $\leq 200{ }^{\circ} \mathrm{C}$. Barium titanate is a normal ferroelectric with the perovskite $\mathrm{ABO}_{3}$ crystal structure that can accommodate different cations on $\mathrm{A}$ and $\mathrm{B}$ sites. By introducing appropriate chemical disorder $\mathrm{BaTiO}_{3}$ changes to a relaxor ferroelectric with a broad frequencydependent peak in the $\varepsilon_{r}-T$ response. At sufficiently high levels of chemical modification, invariably involving bismuth substitution on A -lattice sites, the relaxor peak becomes suppressed and a near stable $\varepsilon_{\mathrm{r}}$ value occurs over a wide temperature range, extending to temperatures well above the operating limit of conventional $\mathrm{BaTiO}_{3}$ based capacitors. This type of material is referred to as a 'temperature-stable relaxor'. Empirical work has shown the effects of compositional complexity in suppressing the relaxor peak in various $\mathrm{BaTiO}_{3}$ based materials. For example, the solid solution in this study, $(1-x) \mathrm{Ba}_{0 .} \mathrm{Ca}_{0.2} \mathrm{TiO}_{3}-(x) \mathrm{BiMg}_{0.5} \mathrm{Ti}_{0.5} \mathrm{O}_{3}$ (from here $\mathrm{BCT}-\mathrm{BMT}$ ), behaves as a normal relaxor when $\mathrm{x}=$ 0.1 , but with increasing BMT content, the $\varepsilon_{\mathrm{r}-\mathrm{T}}$ peak becomes broader and at $x=0.5$ ( or 0.55 ), temperature stable relaxor behaviour is observed, with a near-plateau in relative permittivity: $\varepsilon_{\mathrm{r}} \sim 1060 \pm$ 40 , over the temperature range, $65-550^{\circ} \mathrm{C}[1]$.

We have used STEM to understand how local crystal structure and nanochemistry controls the stability of the relative permittivity over such a wide temperature range in order to provide a fundamental understanding of the mechanisms that convert normal to temperature stable relaxor behaviour. Ceramics were produced by a conventional mixed oxide route. The calcined powders were converted into pellets and sintered in alumina crucibles at $1050{ }^{\circ} \mathrm{C}$ for 4 hours [1]. Samples were prepared for electron microscopy in different ways: 1) crushing the pellet in an agate mortar until a fine powder was obtained, followed by suspension in ethanol and sonication, before dropcasting onto a holey carbon film supported on a 400 mesh copper grid (EM resolutions Ltd); 2) preparing a thin lamella using a Focused Ion Beam FEI Helios G4 CX Dual Beam microscope and in-situ-lift out onto dedicated support grids for TEM (Omniprobe Inc). Samples were analyzed by a FEI Titan Themis ${ }^{3}$ G2 transmission electron microscope operated at $300 \mathrm{kV}$ with a monochromator, a Super-X 4-detector silicon drift energy dispersive X-ray (EDX) system and a Gatan GIF Quantum 965 electron energy loss spectrometer (EELS). STEM was performed with a $1.4 \AA$ probe diameter of $10 \mathrm{mrad}$ convergence semi angle; probe currents were varied by the monochromator and ranged from $40-200 \mathrm{pA}$ depending on the imaging and mapping mode. STEM-EEL spectra were collected with an 11 mrad collection semi-angle. EDX spectra were processed in Bruker Esprit Software and EELS processed in Gatan Microscopy Suite version 3.3.1. On increasing 
the $\mathrm{Bi}$ and $\mathrm{Mg}$ content, $x$, in the (1-x)BCT- $x \mathrm{BMT}$ solid solution, the material changes from a normal relaxor $(x=0.1)$ to an intermediate state $(x=0.4)$ to a temperature stable relaxor $(x=0.55)$ (Figure 1$)$. Atomic column resolution images of the perovskite lattice were obtained by relatively low current (40 pA) HAADF-STEM imaging of the $x=0.1, x=0.4$ and $x=0.55$ powders of crushed pellets (Figure 1). As increasing levels of $\mathrm{Bi}$ and $\mathrm{Mg}$ (from BMT) are incorporated into the crystal structure, chemical inhomogeneity becomes evident across the BCT-BMT lattice. STEM energy dispersive X-ray (EDX) and EELS mapping of the $x=0.55$ temperature stable relaxor, revealed significant variations in concentration of $\mathrm{Ba}, \mathrm{Ti}$, and $\mathrm{Bi}$ over length scales of $\sim 3$ to $5 \mathrm{~nm}$ [2]. The chemical variability between the ferroelectric ( $\mathrm{Ba} / \mathrm{Ti}$ rich) and weakly polar ( $\mathrm{Bi}$ rich) nanoclusters identified in this work for a material with near-flat $\varepsilon_{\mathrm{r}}-\mathrm{T}$ response (above $\mathrm{T}_{\mathrm{m}}$ ) is proposed to inhibit the development of polar coherence on cooling below the Burns temperature that normally occurs in a relaxor ferroelectric with distinct $\varepsilon_{\mathrm{r}}$-T peak (as in the case of $x=0.1$ which did not display evidence of nanosegregation) [3].

\section{References:}

[1] A Zeb and SJ Milne, J. Am. Ceram. Soc. 96 (2013), p. 2887.

[2] T Roncal-Herrero et al., Acta Materialia 158 (2018), p. 422.

[3] Work funded by the U.K. Engineering and Physical Sciences Research Council (EP/P015514/1).

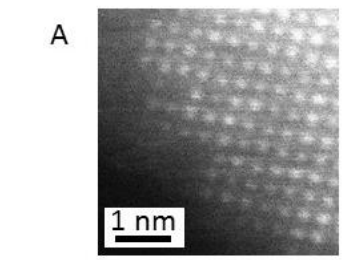

$x=0.1$
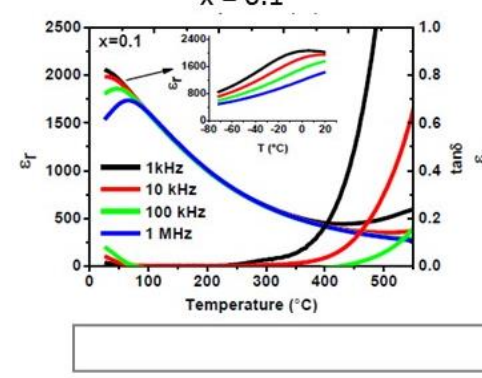

B

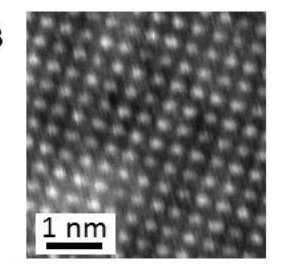

$x=0.4$

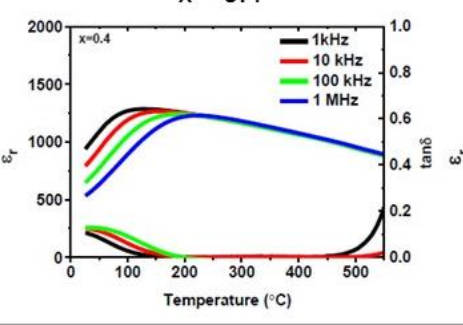

$\mathrm{Bi}\left(\mathrm{Mg}_{0.5} \mathrm{Ti}_{0.5}\right) \mathrm{O}_{3}$

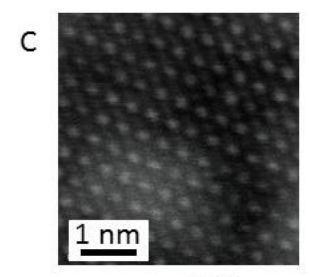

$x=0.55$

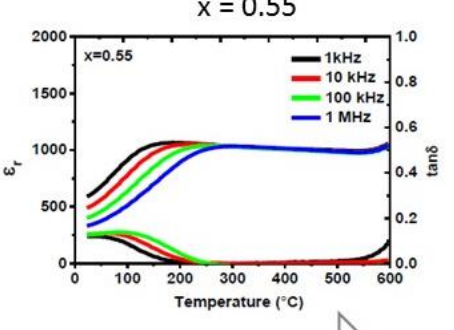

(1)

Figure 1. Low current HAADF-STEM imaging of crushed pellets and the corresponding dielectric properties of $(1-\mathrm{x})\left(\mathrm{Ba}_{0.8} \mathrm{Ca}_{0.2}\right) \mathrm{TiO}_{3}-\mathrm{xBi}\left(\mathrm{Mg}_{0.5} \mathrm{Ti}_{0.5}\right) \mathrm{O}_{3}$ for $\left.\left.\mathrm{a}\right) \mathrm{x}=0.1, \mathrm{~b}\right) \mathrm{x}=0.4$ and $\left.\mathrm{c}\right) \mathrm{x}=0.55$.
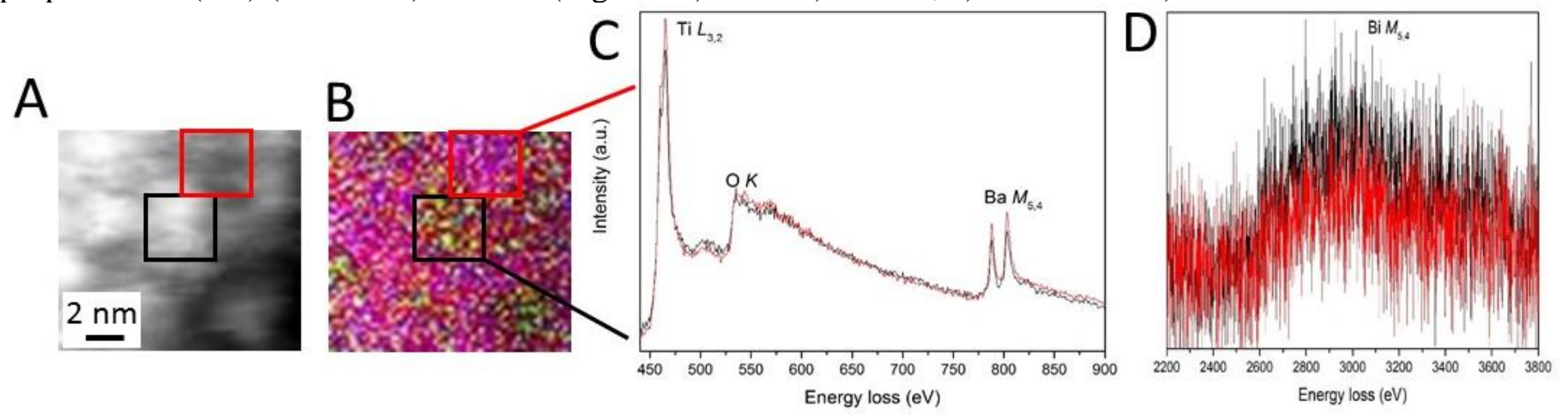

Figure 2. a) HAADF image of (1-x) $\left.\left(\mathrm{Ba}_{0.8} \mathrm{Ca}_{0.2}\right) \mathrm{TiO}_{3}-\mathrm{xBi}\left(\mathrm{Mg}_{0.5} \mathrm{Ti}_{0.5}\right) \mathrm{O}_{3} \mathrm{x}=0.55 \mathrm{~b}\right)$ false color EELS elemental mapping of the same area as a) $\mathrm{Ti} L_{3,2}$ (red), $\mathrm{Ba} M_{5,4}$ (blue) and $\mathrm{Bi} M_{5,4}$ (green) c) and d) background subtracted energy loss edges extracted from the black and red boxes in ( $a$ and $b$ ). 